Briggs, S., Crawford, K., Abraham, E. P. \& Gladstone, G. P. (1957). J. gen. Microbiol. 16, 614-627

\title{
Some Properties of Gram-negative Bacilli Obtained from a Strain of Staphylococcus aureus in the Presence of Benzylpenicillin
}

\author{
By SHIRLEY BRIGGS, KATHLEEN CRAWFORD, \\ E. P. ABRAHAM AND G. P. GLADSTONE \\ Sir William Dunn School of Pathology, University of Oxford
}

\begin{abstract}
SUMMARY: Gram-negative bacilli were obtained from the Oxford strain of Staphylococcus aureus in the presence of benzylpenicillin, and single organisms were isolated from these bacilli. The properties of bacilli derived from the isolates were compared with those of the parent staphylococci. The two types of organism differed strikingly in morphology, staining and various biochemical reactions. The bacilli were much more resistant to benzylpenicillin than the parent staphylococcus, but much less resistant to polymyxin. Staphylococci were recovered from all the strains of bacilli. More than half of the recovered strains resembled the original staphylococcus in all properties tested, including phage type, when first isolated. The remainder formed a somewhat heterogeneous group, differing in some properties from the parent. Later, some members of this heterogeneous group also became indistinguishable from the original staphylococcus.
\end{abstract}

Changes in the morphology of bacteria grown in the presence of subinhibitory amounts of penicillin were first observed by Gardner (1940). He found that staphylococci and streptococei became swollen, and that a number of bacilli formed long filaments. Many similar observations were made subsequently by others (see Florey et al. 1949). In 1944, Rake, McKee, Hamre \& Houck reported that strains of Staphylococcus aureus which had acquired resistance to penicillin contained a proportion of organisms that did not retain the Gram stain. Klimek, Cavallito \& Bailey (1948) stated that a strain of $S$. aureus which was subcultured many times in the presence of increasing amounts of penicillin and developed a very high resistance to the drug eventually became Gram-negative and bacillary in form.

In recent experiments Crawford \& Abraham (1957) found that Gramnegative bacilli could be obtained from Staphylococcus aureus during one culture in the presence of benzylpenicillin, cephalosporin $\mathrm{C}$, or bacitracin. The present paper describes a further study of the bacilli formed in the presence of benzylpenicillin. The strain of $S$. aureus used was derived from a single organism; this was grown in medium containing benzylpenicillin, and several singlecell isolations were made from the Gram-negative organisms that were obtained.

\section{METHODS}

\section{Materials and methods of culture}

Benzylpenicillin, 1650 units/mg. (Glaxo Laboratories Ltd.), was used as the crystalline sodium salt. Polymyxin B sulphate (7909 units/mg.) was kindly provided by the Wellcome Research Laboratories. The medium was in most 
cases a tryptic heart digest broth (TMB) or a tryptic heart digest agar (TMA). Aerated cultures were grown in inverted T-tubes as described by Crawford $\&$ Abraham (1957). Anaerobic cultures were grown in tubes of freshly boiled TMB incubated in an anaerobic jar at $37^{\circ}$. Twofold serial dilution tests were used for measuring antibacterial activity.

Staphylococcus aureus (Oxford strain, NCTC 6571) was the parent organism from which all others were derived.

\section{Methods used in the recognition of the Oxford staphylococcus}

(1) Fermentation tests. Solutions of $1 \%(\mathrm{w} / \mathrm{v})$ lactose, glucose, maltose, mannitol and sucrose in peptone water were used, with phenol red as indicator.

(2) Growth on salt agar. The medium used was $10 \%(\mathrm{w} / \mathrm{v}) \mathrm{NaCl}$ in TMA.

(3) Growth on penicillin agar. Benzylpenicillin was added to TMA at almost $50^{\circ}$ to give a final concentration of $1 \mu \mathrm{g} . / \mathrm{ml}$.

(4) Production of protease. Plates of $1 \%(\mathrm{w} / \mathrm{v})$ gelatin in Difco heart infusion agar were inoculated, incubated at $37^{\circ}$, and then flooded with $15 \%$ $(\mathrm{w} / \mathrm{v}) \mathrm{HgCl}_{2}$ in $\mathrm{N}-\mathrm{HCl}$. Protease production was shown by a clear zone round the inoculum.

(5) Production of coagulase. Fresh human plasma was diluted 1/10 in TMB and the resulting solution inoculated with one loopful of an overnight TMB culture, and then incubated at $37^{\circ}$ overnight.

(6) Production of haemolysins. To detcet $\alpha$-haemolysin rabbit red blood cells were washed 3 times with saline and suspended in a volume of saline equal to the original volume of blood and plates poured with TMA mixed at $c .45^{\circ}$ with $\mathbf{2 . 5} \%(\mathrm{v} / \mathrm{v})$ of the red cell suspension. Half the plate surface was then covered with $0.1 \mathrm{ml}$. of antiserum containing 150 units of $\alpha$-antitoxin (obtained from Mrs I. Batty of the Wellcome Research Laboratories, Beckenham). Cultures were inoculated on both halves of the plate and incubated at $37^{\circ}$ in a mixture $(\mathrm{v} / \mathrm{v})$ of $20 \% \mathrm{CO}_{2}+80 \%$ air. The production of a diffuse-edged lysed zone on the side of the plate not covered with antitoxin and the absence of any zone on the side covered with antitoxin indicated the presence of $\alpha$-haemolysin.

$\delta$-Haemolysin was detected in the following way. Plates were poured as described for $\alpha$-haemolysin except that washed horse blood cells were used in place of rabbit cells. The plates were incubated in air at $37^{\circ}$ after inoculation. A sharp-edged lysed zone round the colonies indicated the presence of $\delta$-haemolysin.

(7) Phage typing. This was carried out by $\operatorname{Dr}$ R. E. O. Williams of the Staphylococcus Reference Laboratory, Colindale, using the standard procedure (Anderson \& Williams, 1956).

(8) Pathogenicity test. Two mice (18-25 g.) were given intraperitoneally $0.5 \mathrm{ml}$. of a just visible suspension of an $18 \mathrm{hr}$. TMA culture. After 10-15 days the animals were killed and examined for lesions in the organs, from which cultures were made.

Sterility precautions and single-cell isolations. The TMB medium was sterilized by autoclaving for $20 \mathrm{~min}$. at $115^{\circ}$ in the culture vessel. All media were incubated for 2 days at $37^{\circ}$, under test conditions, and then for 2 or more 
days at room temperature before inoculation. Benzylpenicillin was sterilized by filtration through a sintered glass bacteriological filter (maximum pore size $1 \cdot 4 \mu$.). Serial subcultivation and any other manipulations involving opening the culture tubes, were done in a tissue-culture box. The box was irradiated with ultraviolet light for $15 \mathrm{~min}$. before each series of manipulations. Uninoculated plates were exposed to the air inside the box during each manipulation, and then incubated at $37^{\circ}$ for 2 days followed by 2 days at room temperature. In no case were any contaminants found. Even so, plates were never used in serial culture work.

To ensure that the cultures used began as pure lines, single-cell isolations were made by a method similar to that of de Fonbrune (1949), by means of a Singer micromanipulator (Barer \& Saunders-Singer, 1951). Single organisms were placed by means of a micropipette in sterile hanging drops of TMA which were suspended in a liquid paraffin oil-cell. The micropipette was attached by pressure tubing to an 'Agla' micrometer syringe modified to draw in and out, and both the pipette and the syringe were filled with paraffin. TMB medium for the hanging-drops was clarified by centrifuging.

Isolation of the single-cell culture $(G+)$ from the Oxford staphylococcus. A T-tube containing $10 \mathrm{ml}$. of TMB and several sterile glass beads was inoculated with the Oxford staphylococcus and shaken for $2 \mathrm{hr}$. at $37^{\circ}$. This treatment gave a suspension of evenly dispersed cocci, most of which were viable. Small drops of this culture were used as reservoirs in the oil-cell from which single organisms were extracted and transferred to hanging-drops of sterile TMB. These single-seeded drops were incubated overnight at $37^{\circ}$, and the isolates which grew extracted with a needle, put on to TMA slopes and incubated for $18 \mathrm{hr}$. at $37^{\circ}$. One of these cultures was designated $\mathrm{G}+$ and used as the parent strain.

\section{RESULTS}

\section{Morphological changes in the Oxford staphylococcus grown in the presence of benzylpenicillin}

Cultures which finally developed from the Oxford staphylococcus in aerated TMB containing benzylpenicillin ( $1 \mu \mathrm{g} . / \mathrm{ml}$.) usually consisted of a mixture of apparently normal staphylococci and Gram-negative rods. On several occasions the Gram-negative forms began to appear during the lytic phase, when the opacity was still falling (cf. Crawford \& Abraham, 1957). More often, however, their appearance was delayed until the opacity was at its lowest point, or was undergoing the secondary increase.

The relative number of the two types of organism in the secondary cultures varied from one experiment to another, but in some cases the proportion of Gram-negative rods was as high as $90 \%$ (Pl. 1, fig. 1). When such mixtures were inoculated into aerated TMB not containing benzylpenicillin they yielded new cultures containing a greatly increased proportion of staphylococci ( $\mathrm{Pl}$. 1, fig. 2). On the other hand, when grown a second time in aerated TMB containing benzylpenicillin they developed into cultures containing mainly long Gram-negative filaments ( $\mathrm{Pl}$. 1, fig. 3). To ensure that this phenomenon 


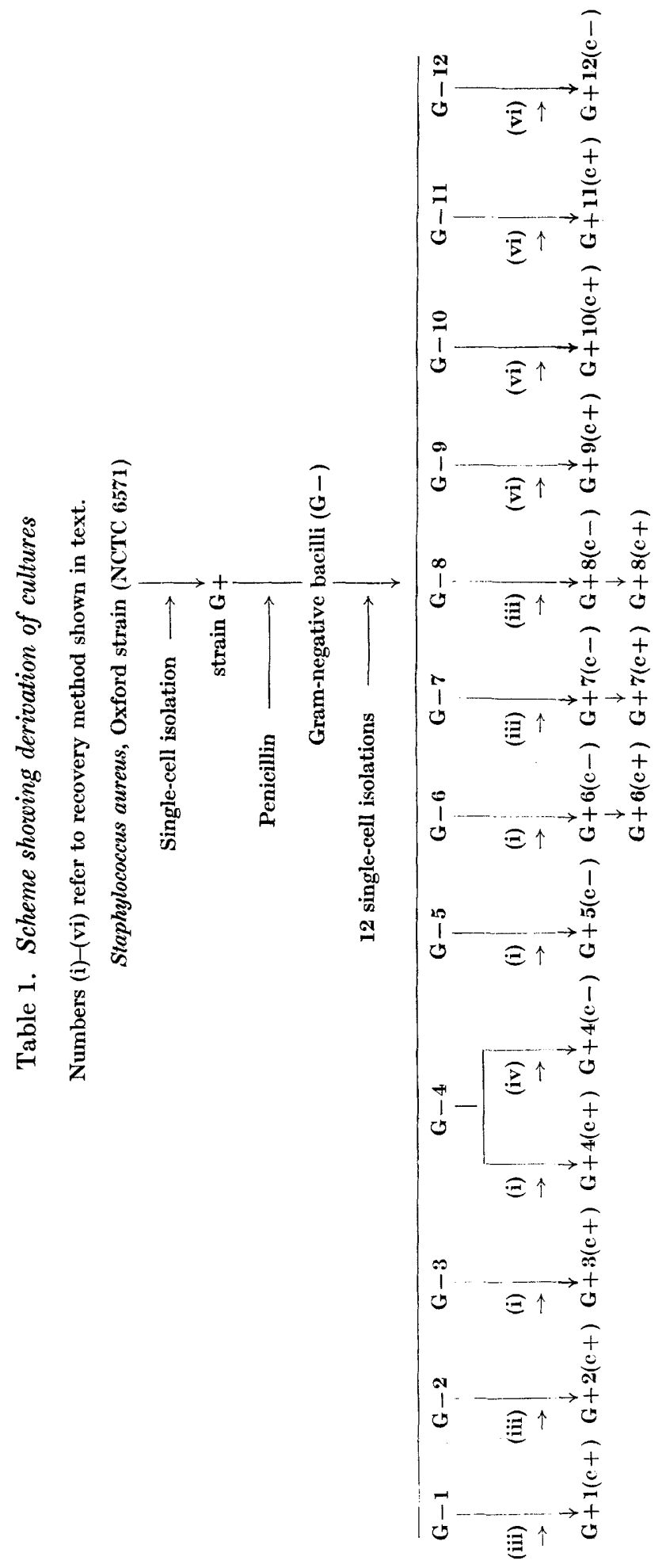


represented a change in the staphylococcus itself further studies were made with cultures derived from single organisms.

The following work was carried out with the sterility precautions described in Methods; Table 1 outlines the relationships between the cultures obtained.

\section{Penicillin treatment of strain $G+$ (the Oxford strain single-cell isolate)}

Benzylpenicillin (final concentration $1 \mu \mathrm{g} . / \mathrm{ml}$.) was added to an aerated culture of $\mathrm{G}+$ growing in a rocking $\mathrm{T}$-tube. The opacity/time curve was of the same type as those shown by Crawford \& Abraham (1957, Fig. 1). The interval between the addition of benzylpenicillin and the start of the final increase in opacity was $22 \mathrm{hr}$. The resulting culture consisted of Gram-negative rods of varying lengths and a few Gram-positive cocci. The T-tube had only been opened twice since sterilization, first to add the inoculum, second to add the benzylpenicillin.

\section{Isolations of Gram-negative rods obtained from strain $G+$ after penicillin treatment}

Single-cell isolations of the Gram-negative rods were made from the culture described in the previous paragraph in the same manner as used to obtain strain $\mathbf{G}+$. Some difficulty was experienced in this selection, as many bacilli failed to grow when seeded singly into sterile drops of TMB. However, when the primary isolate was incubated in air enriched with $5 \%(\mathrm{v} / \mathrm{v}) \mathrm{CO}_{2}$, the number of successful isolations was considerably increased. Twelve isolates, designated $G-1$ to $G-12$, were put on to TMA slopes and on to slopes containing $1 \mu \mathrm{g}$. benzylpenicillin $/ \mathrm{ml}$. and incubated for $18 \mathrm{hr}$. at $37^{\circ}$.

\section{Attempts to recover the original staphylococcus, strain $G+$, from the cultures $G-1$ to $G-12$}

The following methods ( $\mathrm{i}$ to vi) were used in attempts to recover the strain $\mathbf{G}+$ from strains $\mathrm{G}-\mathbf{1}$ to $\mathbf{G}-\mathbf{1 2}$.

(i) The Gram-negative organisms were grown under anaerobic conditions for 2 days in TMB, after a series of eight daily subcultures in TMB.

(ii) They were subcultured daily in TMB, or on TMA slopes.

(iii) They were subcultured at two-weekly intervals, from TMA slopes which had been first incubated at $37^{\circ}$ for $18 \mathrm{hr}$. and then left for the remainder of the 2 weeks at room temperature.

(iv) A series of eight daily subcultures in TMB or on TMA was followed by a number of subcultures in the deficient medium used by Bellamy \& Klimek (1948). This medium was Seitz-filtered and incubated for 3 days before inoculation from an $18 \mathrm{hr}$. TMA slope.

(v) After two subcultures in TMB the Gram-negative organisms were subcultured twice in aerated TMB containing $10 \%(\mathrm{w} / \mathrm{v}) \mathrm{NaCl}$. The final culture was diluted (1/10) with TMB to give a salt concentration of $1 \%$. This diluted culture was incubated at $37^{\circ}$ for $18 \mathrm{hr}$., a loopful extracted and transferred to a TMA slope.

(vi) The Gram-negative organisms were subjected to intermittent sub- 
culture on TMA slopes, for about 4 months, interspersed with periods of several weeks on the bench.

The final step in each method of isolating the Gram-positive coccus from the Gram-negative bacillus was selection on salt agar slopes, and not by plating. The possibility of an adventitious Gram-positive coccus contaminating the culture was thus reduced to negligible proportions.

The methods described above are a rather arbitrary combination of serial subculture and procedures selective for the growth of staphylococci. Nevertheless, Gram-positive cocci were recovered from eight of the twelve G-isolates $(G-1$ to $G-8)$ by one or more of methods (i) to (v), of which those involving anaerobic culture and culturing at intervals of 2 weeks proved most successful, G-9 to G-12 remained unaltered under these conditions but yielded Grampositive cocci by method (vi).

The results are shown in Table 1 , in which Gram-positive strains used in further tests are designated $\mathbf{G}+\mathbf{1}(\mathbf{c}+)$ etc., where $\mathbf{G}+\mathbf{1}(\mathbf{c}+)$ was obtained from $\mathrm{G}-1$ by method (iii) and was coagulase-positive, and where $\mathrm{G}+4(\mathrm{c}+)$ and $G+4(c-)$ were obtained from $G-4$ by methods (i) and (iv) respectively and were coagulase-positive and coagulase-negative respectively.

\section{Comparison of strain $G+$ with strains $G-1$ to $G-12$ and the recovered strains $G+1(c+)$ to $G+12(c-)$}

The Gram-negative strains $G-1$ to $G-12$ were alike in all properties considered in this section, but contrasted dramatically with the parental strain $\mathrm{G}+$. The staphylococci recovered from these strains may be divided into two groups, consisting of strains apparently identical with the original $\mathbf{G}+$, and strains differing in some properties from strain $\mathbf{G}+$.

Cultural properties in liquid media. The parent strain $\mathrm{G}+$ grew vigorously in aerated TMB, well in stagnant TMB, and more slowly in anaerobic TMB. Strains $G-1$ to $G-12$ grew very rapidly in aerated TMB, but less vigorously in stagnant TMB, most of the growth forming an easily dispersed pellicle on the surface of the broth. In anaerobic TMB G-1 to G-12 grew very poorly (see attempts to recover $\mathbf{G}+$ by Method (i)). The growth of all the recovered Gram-positive strains in aerated or anaerobic liquid culture was very similar to that of strain $\mathbf{G}+$.

Cultural properties on solid media. Strain G+ grew well on TMA, forming regular, opaque, cream-white butyrous colonies (see white colonies in $\mathrm{Pl}$. 1, fig. 4). Strains $G-1$ to $G-12$ also grew well on TMA, forming regular and somewhat translucent, smooth, slightly mucoid, yellowish white colonies (see translucent colonies--Pl. 1, figs. 4, 6). Growth on $1 \mu \mathrm{g}$. benzylpenicillin $/ \mathrm{ml}$. in TMA was good, and here the type of colony formed was variable. Young colonies obtained from Gram-negative rods which had been freshly isolated from strain G + often had a 'speckled' appearance (Pl. 1, fig. 5). Incubation for a further $18 \mathrm{hr}$. produced a smoother growth. Sometimes the colonies on benzylpenicillin + TMA were smooth, especially when the strain had been subcultured several times after the original isolation, but such colonies were occasionally sectored by wedges of the 'speckled' growth (Pl. 2, fig. 7). 
The colonies of the recovered strains, $G+1(c+), G+2(c+), G+3(c+)$, $\mathrm{G}+4(\mathrm{c}+), \mathrm{G}+4(\mathrm{c}-), \mathrm{G}+9(\mathrm{c}+), \mathrm{G}+\mathbf{1 0}(\mathrm{c}+)$ and $\mathrm{G}+11(\mathrm{c}+)$, when grown on TMA resembled those of strain $G+$ in colour and opacity, whereas those of strains $\mathrm{G}+5(\mathrm{c}-), \mathrm{G}+7(\mathrm{c}-), \mathrm{G}+8(\mathrm{c}-)$ and $\mathrm{G}+12(\mathrm{c}-)$ differed in being more opaque and whiter, and strain $G+6(c-)$ had a slightly pinkish tinge.

Morphological properties. The $\mathbf{G}+$ organisms were those of a typical staphylococcus with cocci regular in shape and size arranged in bunches (in hangingdrop cultures) and staining Gram-positive (see cocci in Pl. 1, fig. 2). In marked contrast, strains $G-1$ to $G-12$ showed organisms varying from very long rods to very short oval forms in the same culture. The rods were usually continuous along their length, but were occasionally in chains of shorter bacilli. They were very pleomorphic, non-motile, predominantly Gramnegative but with occasional thickenings, lumps and club-shaped distortions of Gram-positive material. The morphology of these organisms altered when they were grown in different media, and for different periods of incubation. In aerated $T M B$ growth of strains $G-1$ to $G-12$ was made up of consistently Gram-negative rods, medium to short in length and fairly uniform in width (see bacilli in Pl. 1, fig. 2). When $1 \mu \mathrm{g}$. benzylpenicillin $/ \mathrm{ml}$. was added, very long rods were formed, again fairly uniform in width (see filaments in Pl. 1, fig. 3). In stagnant TMB medium a pellicle was formed which consisted of rods of varied shape and length, often with Gram-positive lumps and patches. After several subcultures in stagnant TMB medium, the rods became shorter and many stained Gram-positive. Eventually, after a series of subcultures it was possible to recover Gram-positive cocci from some of the single-cell isolates (see attempts to recover strain $\mathrm{G}+$ by Method (ii)). In anaerobic conditions the rods did not grow as such, but as distorted Gram-positive cocco-bacilli (see attempts to recover strain $\mathbf{G}+$ by Method (i)). On solid media colonies were made up of Gram-negative rods showing a wide range of forms. These varied from very long, exceedingly pleomorphic filaments, when grown on $1 \mu \mathrm{g}$. benzylpenicillin/ml. in TMA (Pl. 2, fig. 8), to very short Gramnegative cocco-bacilli (Pl. 2, fig. 12), when subcultured on TMA without penicillin. There were intermediate types with different proportions of long, medium and short rods (Pl. 2, figs. 9-11). The proportion of short cocco-bacilli increased with longer incubation and with more subcultures without penicillin. Over-all, a well-aerated medium containing penicillin seemed to provide the best conditions for the most extravagant forms of long bacillus.

The cocci of all the recovered $\mathrm{G}+$ strains resembled those of the original strain $\mathrm{G}+$ in size, regularity of shape, arrangement and Gram-staining.

Sensitivity to benzylpenicillin and polymyxin $B$. Dilution tests were used to determine the sensitivity of some of the organisms to benzylpenicillin and to polymyxin $\mathrm{B}$. The concentrations of these antibiotics which were inhibitory to the growth of representative strains for 1 and 4 days respectively are shown in Table 2. It will be seen that strains $G+, G+2(c+), G+3(c+)$ and $G+6(c-)$ were of the same order of sensitivity to benzylpenicillin, while strains $G-3$ and $\mathrm{G}-12$ were extremely resistant. Moreover, with the Gram-negative strains the end-point was not clear-cut, and there were traces of growth even at 
concentrations as high as $10,000 \mu \mathrm{g}$. benzylpenicillin $/ \mathrm{ml}$. In contrast, the Gram-negative organisms were much more sensitive to polymyxin than the Gram-positive ones.

\section{Table 2. Sensitivity to penicillin and polymyxin of staphylococci and Gram-negative variants}

The organisms are named according to the code given in Table 1.

\begin{tabular}{|c|c|c|c|c|}
\hline \multirow[b]{2}{*}{ Organism } & \multicolumn{2}{|c|}{$\begin{array}{l}\text { Concentration of } \\
\text { penicillin }(\mu \mathrm{g} \cdot / \mathrm{ml} .) \\
\text { inhibitory for }\end{array}$} & \multicolumn{2}{|c|}{$\begin{array}{c}\text { Concentration of } \\
\text { polymyxin } \mathrm{B}(\mu \mathrm{g} . / \mathrm{ml} .) \\
\text { inhibitory for }\end{array}$} \\
\hline & 1 day & 4 days & 1 day & 4 days \\
\hline $\mathbf{G}+$ & 0.03 & 0.06 & 50 & 100 \\
\hline$G+2(c+)$ & $0 \cdot 015$ & 0.015 & 50 & 100 \\
\hline $\mathbf{G}+\mathbf{3}(\mathbf{c}+)$ & 0.06 & 0.12 & 50 & 50 \\
\hline$G+4(c-)$ & • & • & 50 & 100 \\
\hline$G+6(c-)$ & 0.03 & 0.06 & . & . \\
\hline $\mathbf{G}-\mathbf{3}$ & 100 & 1000 & 0.78 & 0.78 \\
\hline$G-11$ & . & . & 0.24 & $0 \cdot 24$ \\
\hline$G-12$ & 50 & 1000 & $0 \cdot 20$ & $0 \cdot 20$ \\
\hline
\end{tabular}

Biochemical properties. The results of Tests 1-6, described in Methods, are shown in Table 3. Strain G + fermented the sugars tested, was able to grow on $10 \% \mathrm{NaCl}$ agar, and produced protease, coagulase and $\alpha$-haemolysin. The $\mathbf{G}$-isolates differed from strain $\mathbf{G}+$ in every one of these tests. Of the recovered strains, $G+1(c+)$ to $G+4(c+)$ and $G+9(c+)$ to $G+11(c+)$ had test reactions identical with those of the original strain $G+$, whereas the remainder did not produce coagulase and differed from strain $\mathrm{G}+$ in several other tests. Strains $G+4(c-)$ and $G+12(c-)$ were unable to ferment mannitol and did not produce protease or $\alpha$-haemolysin. The remaining four strains were also unable to ferment mannitol, and produced in addition a different haemolysin, namely $\delta$-haemolysin. Of these, only strain $\mathrm{G}+5(\mathrm{c}-)$ produced protease, and only strain $\mathrm{G}+8(\mathrm{c}-)$ was unable to ferment lactose.

Phage typing. Dr R. E. O. Williams reported as follows: 'The parent strain, $\mathrm{G}+$, was susceptible to phages, 7, 52, 52 A, 79 and 80 when used 1000 times stronger than the routine test dilution; all other tests were therefore made with the strong phage filtrates. Seven of the recovered staphylococci had phage sensitivity patterns that were practically identical with the parent strain (Table 3); these were the seven that produced coagulase and $\alpha$-haemolysin. The other six strains were untypable. None of the cultures that consisted entirely of Gram-negative bacilli was lysed by the phages. The supernatant fluids of cultures of six representative strains $(G+1(c+), G+2(c+), G+4(c+)$, $G+4(c-), G-6, G-7)$ were tested for the presence of phage using five strains of phage group 1 as indicators. The $G+(c+)$ strains all carried a phage lysing the same indicator strain, which had, in routine typing, the phage sensitivity pattern $52 \mathrm{~A} / 79$. No phage could be found in the supernatants of the untypable strains.'

Pathogenicity tests. These tests were carried out with strains $\mathrm{G}+, \mathrm{G}-\mathbf{2}, \mathrm{G}-\mathbf{3}$, $\mathrm{G}-6, \mathrm{G}-7, \mathrm{G}-11, \mathrm{G}-12, \mathrm{G}+2(\mathrm{c}+), \mathrm{G}+3(\mathrm{c}+), \mathrm{G}+6(\mathrm{c}-)$ and $\mathrm{G}+7(\mathrm{c}-)$. No 


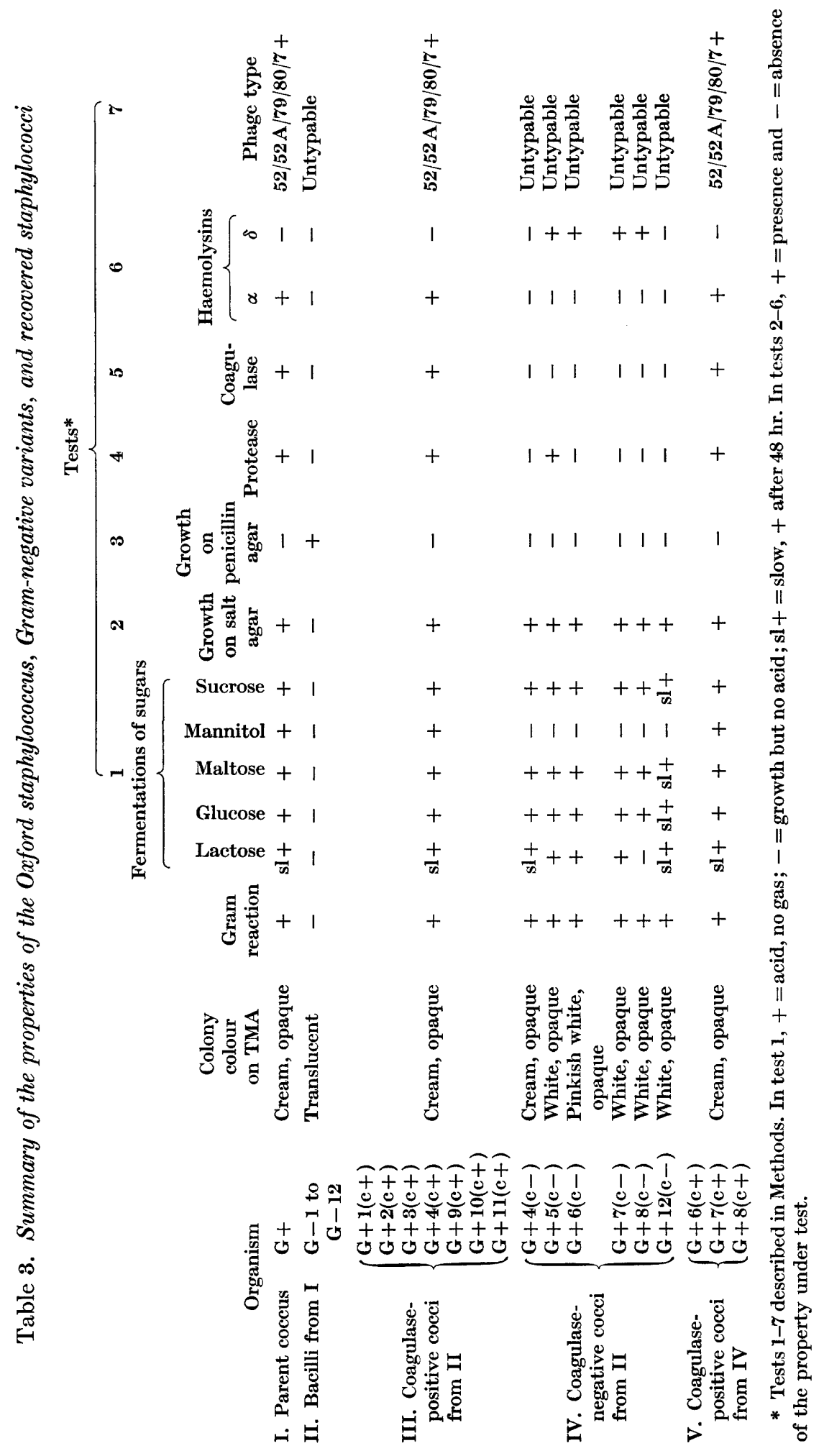


deaths occurred in the mice injected and they were killed after 13 days. No lesions were found with the $\mathbf{G}$ - strains, or with either of the two coagulasenegative strains $\mathrm{G}+6(\mathrm{c}-)$ and $\mathrm{G}+\boldsymbol{\gamma}(\mathrm{c}-)$. The coagulase-positive strains $\mathrm{G}+$, $G+2(c+)$ and $G+3(c+)$ produced large abscesses in the body wall, from which the same strain was recovered. No lesions were found elsewhere.

\section{Change of coagulase-negative (c-) strains of staphylococci to parent type}

Subsequently, after about 4 months of subculture and storage, it was found that three of the coagulase-negative strains $(G+6(c-), G+7(c-)$, and $\mathrm{G}+8(\mathrm{c}-))$ had reverted to coagulase-positive strains with the same phage type as the parent strain $\mathbf{G}+$. The change in properties can be seen in Table 3 . The nature of this reversion is being investigated.

Thus, Gram-positive cocci were recovered from each of the twelve Gramnegative single-cell bacillary isolates. Ten of these recovered strains were identical with the original strain $\mathbf{G}+$, seven being identical when first isolated and three passing through an intermediate stage in which they differed from the parent in several characters.

\section{Other properties of pure-line staphylococci and Gram-negative variants}

Growth rates. The rate of growth of strains $\mathrm{G}+, \mathrm{G}-7$ and $\mathrm{G}+3(\mathrm{c}+)$ was compared in aerated TMB by measuring changes in opacity, the initial opacity being about $\mathbf{0 \cdot 2}$ on the scale used. No significant differences in growth rate were observed. A mixture of strains $\mathbf{G}-\mathbf{7}$ and $\mathbf{G}+$ and another of strains $\mathbf{G}-\mathbf{7}$ and $\mathrm{G}+3(\mathrm{c}+)$ (containing about $50 \%$ of each strain) grew at the same rate as the single strains alone. No change in the composition of the mixtures was observed during growth.

Production of penicillinase. A 5-day culture of the Gram-negative strain G-3 in TMB was kept at $37^{\circ}$ with benzylpenicillin $(1 \mathrm{mg} . / \mathrm{ml}$.) for $16 \mathrm{hr}$. A solution of benzylpenicillin in water $(1 \mathrm{mg} . / \mathrm{ml}$.) was used as a control. No difference was detected in the final activity of the two solutions. In a similar experiment, in which the penicillinase-producing strain D3R was used, complete loss of penicillin occurred in the presence of a 5-day culture.

\section{DISCUSSION}

The present work, in which single cocci of the Oxford staphylococcus were used and exceptional precautions were taken to avoid contamination, leaves no doubt that Gram-negative filaments were really formed from staphylococci during the growth of a single culture in the presence of benzylpenicillin and that they were able to revert to the parent strain. The reason why they were obtained so much more rapidly than in the experiments with penicillin previously described by others is uncertain, but it may be connected with the use of large inocula and aerated cultures. In serial dilution tests, which were carried out in stagnant media, cultures which grew up in the presence of the 
antibiotics always consisted of Gram-positive cocci. Klimek et al. (1948) reported that Gram-negative organisms obtained from Staphylococcus aureus in the presence of penicillin were unable to grow anaerobically. These organisms had also lost the ability to ferment a number of simple carbohydrates, although they were still able to ferment glucose; Gale \& Rodwell $(1948,1949)$ showed that their Gram-negative organisms derived from staphylococci had lost the power of concentrating free glutamic acid within the cells, and had become able to synthesize all their amino acid requirements from ammonia and glucose in the presence of thiamine.

The mixed cultures obtained when the Oxford staphylococcus was grown once in aerated medium containing cephalosporin $\mathrm{C}$, benzylpenicillin, or bacitracin reverted to cultures consisting almost entirely of normal staphylococci during one subculture in aerated medium devoid of antibiotic (Crawford \& Abraham, 1957). Our pure-line Gram-negative variants did not undergo noticeable reversion under these conditions, but they reverted to staphylococci in old colonies on solid media or when subcultured under anaerobic conditions. Klimek et al. (1948) and Bellamy \& Klimek (1948) noted that staphylococci with a moderate degree of resistance to penicillin reverted readily to a sensitive strain, but that cultures which had a very high resistance were more stable. It seems that the Gram-negative variants which are first formed are less stable than their progeny. However, it is not certain whether the reversion of the early mixed cultures is due to a change of Gramnegative organisms to staphylococci or to an ability of the staphylococci to grow faster than these Gram-negative organisms in the absence of antibiotic. Although the growth rate of a pure-line Gram-negative variant did not differ greatly from that of the original staphylococcus in aerated TMB, this was not necessarily the case with the variants that were first produced.

The pure-line Gram-negative variants differed strikingly from staphylococci, not only in morphology and in resistance to certain antibiotics, but also in a number of biochemical properties. They failed to ferment five different sugars, did not liquefy gelatin, produced neither coagulase nor haemolysins, and were resistant to all phages used to type coagulase-positive staphylococci. Some of the staphylococci recovered from these variants were identical with the original Oxford strain in all the properties tested, including phage type. Others, however, had failed to regain the ability to form coagulase and $\alpha$-haemolysin, to ferment mannitol or to liquefy gelatin, and could not be typed by phage. Eventually, some of the latter reverted to the parent type. The formation of the Gram-negative variants may also be a multi-stage process. It is well known, for example, that staphylococci can acquire a high resistance to benzylpenicillin without losing their ability to retain the Gram stain (Klimek et al. 1948; Kawamata \& Shigeto, 1956). It has also been reported that a penicillinase-producing strain of Staphylococcus aureus can give rise, in the presence of penicillin, to cocci which no longer produce penicillinase, are coagulase-negative and fail to ferment mannitol or liquefy gelatin (Voureka, 1952).

Very little information is available, from these experiments, about the 
mechanisms by which such striking changes in the staphylococcus occur. During the initial period of lysis in aerated cultures most of the original staphylococci are certainly killed, the relative decrease in opacity being much less than the decrease in number of viable organisms. Gram-negative forms were sometimes observed during lysis, although the full secondary growth did not occur until much later. Although selection clearly plays a role in the development of the cultures of Gram-negative organisms and in the production, from the latter, of cultures of staphylococci, the hypothesis that all the changes are initiated by spontaneous, independent, mutations is not free from difficulty. A considerable number of different mutations, each of some selective value in the same environment, would seem to be required and the reversal of all of them would be necessary to account for the recovery of what appears to be the original staphylococcus. It is difficult to believe that changes in all the properties tested are associated with differences in growth rate in the presence and absence of benzylpenicillin. Induced mutations, akin to that which has been reported to be responsible, under some conditions, for the development of resistance to streptomycin (Szybalski, 1955), would also need to be readily reversible. These difficulties are not greatly minimized by the fact that some of the cultures of recovered staphylococci were at one stage distinguishable from the parent strain. The hypothesis that a single genetic change is responsibie, directly or indirectly, for changes in a number of different properties, is complicated by the fact that the recovered staphylococci were heterogeneous.

It seems possible, therefore, that some, at least, of the changes observed in the present work occurred without loss of genetic continuity and resulted from the effect of the environment on extranuclear structures. The formation of the Gram-negative bacilli might involve adaptive changes in slowly growing organisms during the lysis of organisms which were growing more rapidly. The cytochemical studies of Boivin, Tulasne, Vendrely \& Minck (1947), which indicated that penicillin interfered with the synthesis of ribonucleic acid and hindered cytoplasmic division without affecting nuclear division, and later work, which has suggested that the primary effect of penicillin is on the synthesis or maintenance of a component of the cell wall (Mitchell, 1957; Cooper, 1956; Lederberg, 1956), may be relevant in this connexion. The finding that a Gram-negative variant was much more sensitive to polymyxin than the original or recovered staphylococcus supports the view that a major change had occurred in the cell wall. Newton (1956) has obtained evidence that the bactericidal activity of polymyxin is a consequence of its ability to damage a membrane which underlies the cell wall and controls osmotic equilibrium. He has suggested that polymyxin is relatively ineffective against most Grampositive bacteria because it cannot readily penetrate their cell walls and reach this sensitive membrane.

During the formation of the Gram-negative bacillary forms the production of active phage is suppressed. The phage is still absent when the bacillary form changes to a coagulase-negative staphylococcus, but reappears when it reverts to a coagulase-positive staphylococcus. The capacity to form phage has been 
reported to control the expression of certain inheritable characters in bacteria -for example, the production of diphtheria toxin (Freeman, 1951), and antibiotics have been shown to alter the character of lysogenic diphtheria bacilli by removal of carried phage (Hewitt, 1954). It is therefore conceivable that the inability of some of the coagulase-negative staphylococci to produce phage is linked with other properties in which these organisms differ from those of the parent strain. This hypothesis, however, would not account for all the facts, because some of the staphylococci which failed to carry phage were not identical in other respects.

We wish to thank Dr R. E. O. Williams for his active co-operation in carrying out investigations into the phage type and lysogenicity of our strains. Our thanks are due to Miss Mary Read and Mrs P. Curtiss for technical assistance and to Mr F. Bradley for photographs. One of us (S. B.) is indebted to the Medical Research Council for a personal grant.

\section{REFERENCES}

Anderson, E. S. \& Williams, R. E. O. (1956). Bacteriophage typing of enteric pathogens and staphylococci and its use in epidemiology: a review. J. clin. Path. 9, 94.

Barer, R. \& Saunders-Singer, A. E. (1951). A low-power micromanipulator and microdissector. J. sci. Instrum. 28, 65.

Bellamy, W. D. \& Klimek, J. W. (1948). Some properties of penicillin-resistant staphylococci. J. Bact. 55, 153.

Boivin, A., Tulasne, R., Vendrely, R. \& Minck, R. (1947). Arch. Sci. Physiol. 1, 307.

Cooper, P. D. (1956). Site of action of radiopenicillin. Bact. Rev. $20,28$.

Crawford, K. \& Abraham, E. P. (1957). The synergistic action of cephalosporin C and benzylpenicillin against a penicillinase-producing strain of Staphylococcus aureus. J. gen. Microbiol. 16, 604.

de Fonbrune, P. (1949). Technique de micromanipulation. Paris: Masson et Cie.

Florey, H. W., Chain, E., Heatley, N. G., Jennings, M. A., Sanders, A. G., Abraham, E. P. \& Florey, M. E. (1949). Antibiotics, chapter 35. Oxford Medical Publications.

Freeman, V. J. (1951). Studies on the virulence of bacteriophage-infected strains of Corynebacterium diphtheriae. J. Bact. 61, 675.

Gale, E. F. \& Rodwell, A. W. (1948). Amino acid metabolism of penicillinresistant staphylococci. J. Bact. 55, 161.

Gale, E. F. \& Rodweli, A. W. (1949). The nature of resistance to penicillin in Staphylococcus aureus. J. gen. Microbiol. 3, $12 \%$.

Gardner, A. D. (1940). Morphological effects of penicillin on bacteria. Nature, Lond. 146, 837.

Hewirt, L. F. (1954). The effect of certain antibiotics and other chemotherapeutic agents on lysogenicity and virulence transfer by bacterial viruses in Corynebacterium diphtheriae. J. gen. Microbiol. 11, 288.

Kawamata, J. \& Shigeto, T. (1956). Altered growth requirements accompanying penicillin resistance. Bull. Hyg. 31, 439.

Klimek, J. W., Cavallito, C. J. \& Bailey, J. H. (1948). Induced resistance of Staphylococcus aureus to various antibiotics. J. Bact. 55, 139.

Lederberg, J. (1956). Bacterial protoplasts induced by penicillin. Proc. nat. Acad. Sci., Wash. 42, 574.

Mitchell, P. (1957). Penicillin and the logic of chemotherapy. First European Symposium on Antibiotics, Milan. G. Microb. (in the Press). 
Journal of General Microbiology, Vol. 16, No. 3

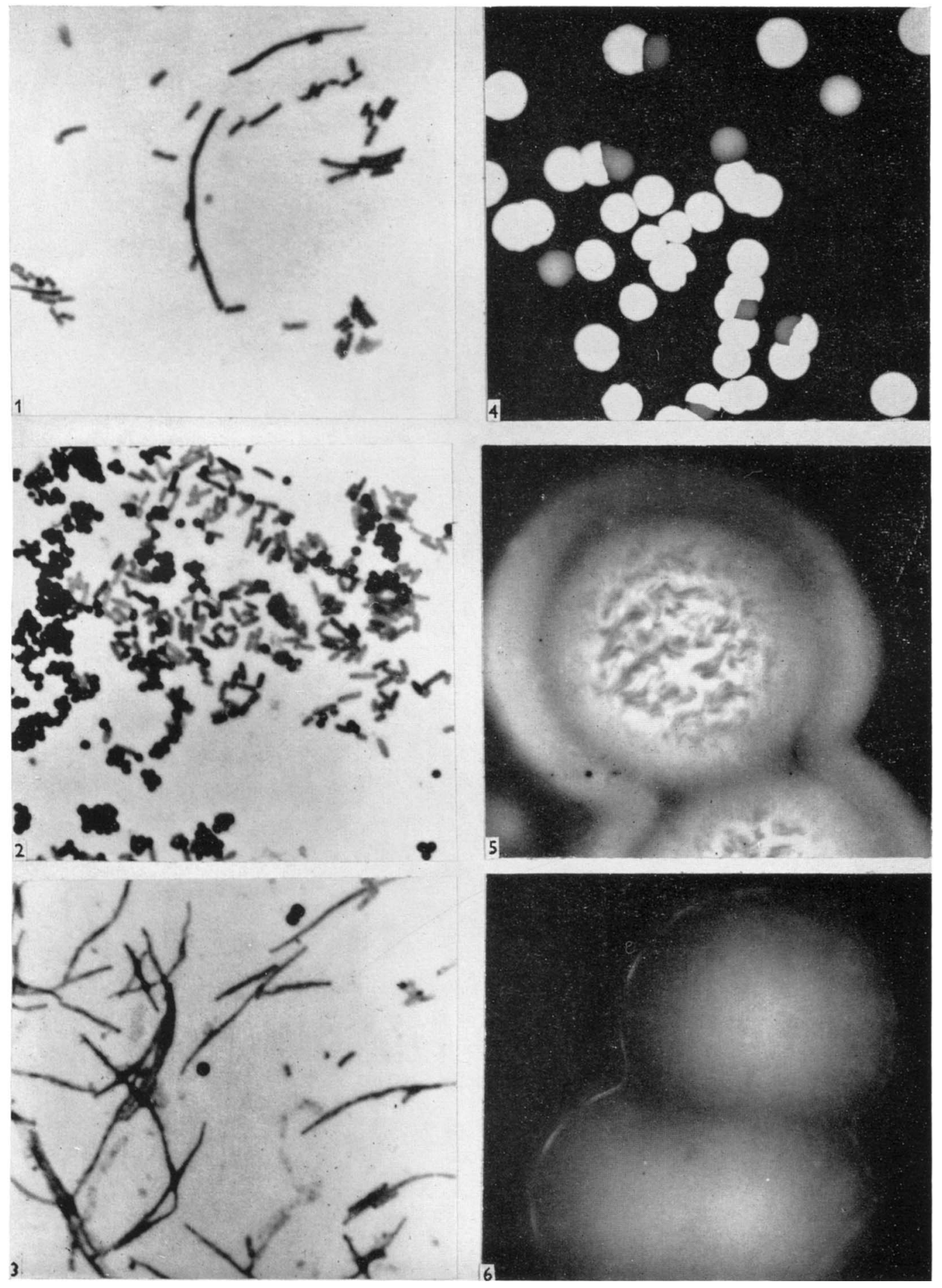

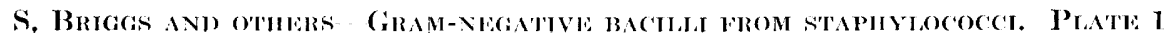

(Facing p. 626) 
Journal of General Microbiology, Vol. 16, No. 3
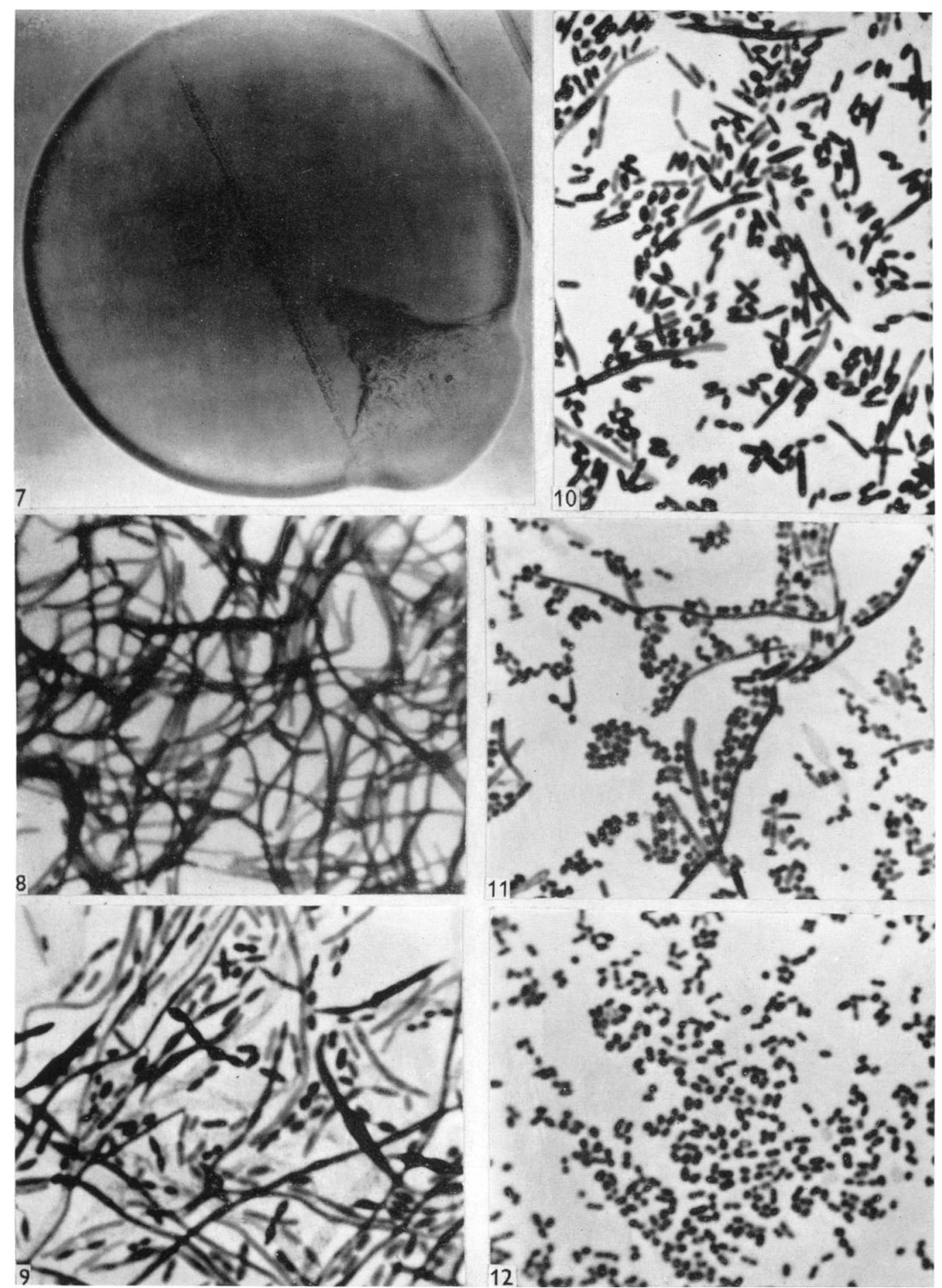

5. bo 2. * $A=\sin ^{2}$ >20. 0 की

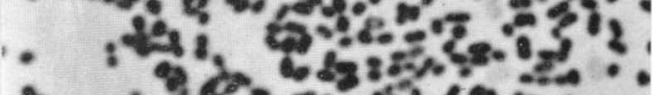

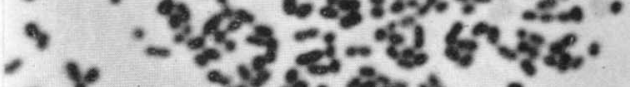
$50-1-8$

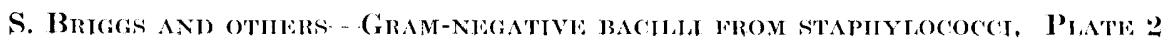


Newton, B. A. (1956). The properties and mode of action of the polymyxins. Bact. Rev. 20, 14.

Rake, G., MCKee, C. M., Hamre, D. M. \& Houck, C. L. (1944). Studies on penicillin. II. Observations on therapeutic activity and toxicity. J. Immunol. 48, 271.

Szybalski, W. (1955). 'Lamarckian' inheritance of streptomycin resistance. Antibiot. Ann. 1954-55.

Votreka, A. (1952). Induced variations in a penicillin-resistant staphylococcus. J. gen. Microbiol. 6, 352.

\section{EXPLANATION OF PLATES}

\section{Plate 1}

Variants of Staphylococcus aureus (single-cell isolate G+, from Oxford strain NC'TC 6571) after treatment with penicillin $(1 \mu \mathrm{g} . / \mathrm{ml}$.). All microscopical preparations were Gram stained (magnification $\times 1500$ ).

Fig. 1. The first growth of Gram-negative bacillary variants in aerated TMB containing penicillin which had been inoculated with $S$. aureus. A few cocei (not shown in the preparation) were still present.

Fig. 2. Subculture of the growth shown in fig. 1, into aerated TMB without penicillin, showing short Gram-negative bacilli and an increased proportion of Gram-positive cocci.

Fig. 3. Subculture of the growth shown in fig. 1, into aerated TMB containing penicillin. The culture is composed mainly of Gram-negative filaments with a few enlarged cocci.

Fig. 4. Colonies of staphylococci (white) and bacillary variants (translucent) on TMA $(\times 2)$.

Fig. 5. Colonial appearance of bacilli grown on TMA containing $1 \mu \mathrm{g} . / \mathrm{ml}$. penicillin for 2 days at $37^{\circ}$, showing the 'speckled' appearance of growth on the first day and a ring of smooth growth which appeared during the second day's incubation $(\times 30)$.

Fig. 6. Colonial appearance of bacilli grown on TMA for 2 days at $37^{\circ}(\times 30)$.

\section{Plate 2}

Fig. 7. 'Smooth' colony of bacilli with a sector of 'speckled' growth, on TMA with penicillin, incubated 2 days at $37^{\circ}$, then left 4 days at room temperature $(\times 30)$.

Fig. 8. Gram-negative filaments from the 'speckled' portion of a colony similar to that shown in Fig. 5 but after incubation for 1 day only.

Fig. 9. Gram-negative filaments derived from a sector of the colony shown in fig. 7 . The sector was transferred to TMA containing penicillin and the plate incubated for 2 days. The Gram-negative filaments were obtained from one of the resulting colonies.

Fig. 10. Bacilli derived from a sector of the colony shown in fig. 7 . In this case part of the sector was transferred to TMA containing no penicillin.

Fig. 11. Bacilli derived from the 'smooth' part of the colony shown in fig. 7. A portion of the colony was plated on TMA containing penicillin and the plate incubated for 1 day. The bacilli were obtained from the resulting colonies.

Fig. 12. Appearance of the Gram-negative variants after 5 subcultures on TMA without penicillin. The organisms are Gram-negative cocco-bacilli.

(Received 16 November 1956) 